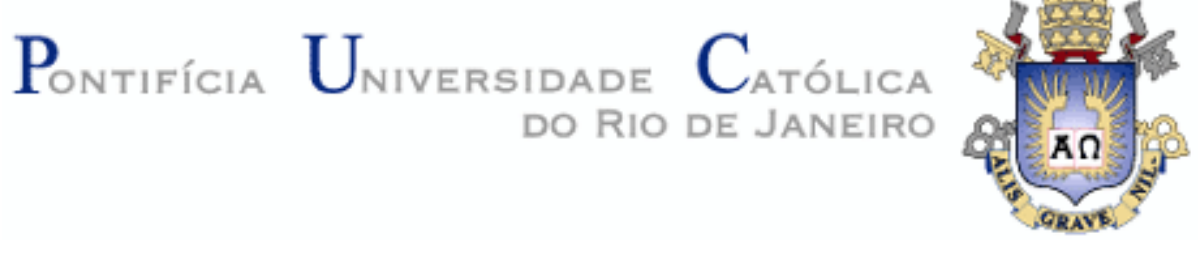

Márcio Thelio Fernandes da Silva

\title{
Projeto \& Desenvolvimento de um padrão para Alta Tensão em Corrente Contínua na Faixa de 1 kV a 50 kV
}

Dissertação apresentada como requisito parcial para obtenção do título de Mestre pelo Programa de Pós-Graduação em Metrologia da PUC-Rio (Área de concentração: Metrologia para Qualidade e Inovação).

Orientador: Prof. Carlos Roberto Hall Barbosa 


\section{Pontifícia Universidade Catállica $_{\text {a }}$}

\section{Projeto \& Desenvolvimento de um padrão para Alta Tensão em Corrente Contínua na Faixa de 1 kV até 50 kV}

Dissertação apresentada como requisito parcial para obtenção do título de Mestre pelo Programa de PósGraduação em Metrologia da PUC-Rio. Aprovada pela Comissão Examinadora abaixo assinada.

Prof. Dr. Carlos Roberto Hall Barbosa

Orientador

Programa de Pós-Graduação em Metrologia (PósMQI) Pontifícia Universidade Católica do Rio de Janeiro (PUC-Rio)

Dr. Fernando Antonio Chagas

CEPEL - Centro de Pesquisas de Energia Elétrica

Dr. João Guedes de Campos Barros

CEPEL - Centro de Pesquisas de Energia Elétrica

Prof. Dr. Reinaldo Castro Souza

Departamento de Engenharia Elétrica (PUC-Rio)

Prof. Dr. Maurício Nogueira Frota

Programa de Pós-Graduação em Metrologia (PósMQI) Pontifícia Universidade Católica do Rio de Janeiro (PUC-Rio)

Prof. José Eugenio Leal Coordenador Setorial de Pós-Graduação do Centro Técnico Científico (PUC-Rio)

Rio de Janeiro, 30 de março de 2011 
Todos os direitos reservados. É proibida a reprodução total ou parcial do trabalho sem autorização da universidade, do autor e do orientador.

\section{Márcio Thelio Fernandes da Silva}

Engenheiro eletricista pela Universidade Gama Filho (1995), pesquisador e responsável pelo Laboratório de Referência em Medição de Alta Tensão do Centro de Pesquisas de Energia Elétrica (CEPEL), desde 2001.

Ficha Catalográfica

Silva, Márcio Thelio Fernandes da
Projeto \& Desenvolvimento de um padrão
para alta tensão em corrente contínua na faixa
de $1 \mathrm{kV}$ a $50 \mathrm{kV} /$ Márcio Thelio Fernandes da
Silva ; orientador: Carlos Roberto Hall Barbosa.
-2010 .
84 f. : il. (color.) ; $30 \mathrm{~cm}$
(mestrado)-Pontifícia
Universidade Caçálica do Rio de Janeiro,
Centro Técnico Científico, 2010.
Inclui bibliografia
1. Metrologia - Teses. 2. Padrão. 3. HVDC.
4. Rastreabilidade. 5. Corrente contínua. 6.
Calibração. 7. Ensaios. I. Barbosa, Carlos
Roberto Hall. II. Pontifícia Universidade Católica
do Rio de Janeiro. Centro Técnico Científico.
Programa de Pós-Graduação em Metrologia
para a Qualidade e Inovação. III. Título.

CDD: 389.1 
Aos meus filhos Thiago e Thaís por reconhecerem na ausência do pai um motivo de orgulho e superação e a minha esposa Tathiana pelo apoio, paciência e compreensão ao longo desse desafio. A eles que sempre me apoiaram, muito obrigado. 


\section{Agradecimentos}

Aos meus pais pela bênção da vida, orientação e educação. Aos meus amados filhos, esposa e amigos que souberam entender minha ausência.

Ao meu amigo e companheiro de trabalho Luiz Carlos de Azevedo, pelo seu apoio incondicional durante toda a fase de estudos e também a toda equipe do laboratório de Referência em Medição de Alta Tensão.

Aos também companheiros de trabalho Orsino Borges de Oliveira Filho e Walter Ramos de Cerqueira Filho, com quem tive a oportunidade impar de trabalhar e pelos preciosos ensinamentos.

Ao amigo e companheiro Alexandre Neves, chefe da DVLF, pelo incentivo a pesquisa como ferramenta de motivação na busca constante por novos desafios.

Aos meus chefes Alain François Sanson Levy da DVLA, Henrique Burd do DPL, pelo apoio institucional. Agradecimentos também são devidos ao Dr. João G. C. Barros, juntamente com o diretor do CEPEL Jorge Nunes de Oliveira, por depositarem sua confiança e incentivarem a transposição desse desafio.

Aos companheiros Athanásio Mpalantinos Neto e Luiz Adriano M C Domingues, pelo apoio na utilização do software para simulações de campos elétricos.

Ao Orientador Professor Doutor Carlos Hall Barbosa, que com seus conselhos e orientação, foi capaz de transformar um sonho em realidade.

A todos os colegas da oficina mecânica do CEPEL, pelo excelente trabalho na confecção das peças e desenhos do padrão.

Aos excelentes professores responsáveis por minha formação e aos funcionários da secretaria do PósMQI, pelo desprendido suporte.

E, finalmente, a Metrologia que entrou na minha vida e não quer mais sair. 


\section{Resumo}

Fernandes da Silva, Márcio Thelio; Hall; Carlos Roberto Barbosa. Projeto \& Desenvolvimento de um padrão para Alta Tensão em Corrente Contínua na Faixa de 1 kV a 50 kV. Rio de janeiro, 2011. 84p. Dissertação de Mestrado - Programa de pós-Graduação em Metrologia (Área de concentração: Metrologia para Qualidade e Inovação), Pontifícia Universidade Católica do Rio de Janeiro.

Metrologia e qualidade estão intimamente relacionadas e constituem-se em poderoso instrumento de transformação da infraestrutura tecnológica de países e organizações. O avanço do desenvolvimento científico e tecnológico está fortemente embasado no avanço da metrologia. O Instituto Nacional de Metrologia, Normalização e Qualidade industrial (INMETRO) é o órgão máximo responsável pela conservação e manutenção das unidades de base e derivadas do Sistema Internacional de Unidades (SI) no país. A grandeza tensão em corrente contínua, até $1 \mathrm{kV}$, está disponível e rastreada a padrões nacionais na Rede Brasileira de Calibração (RBC). Contudo, para tensões maiores que $1 \mathrm{kV}$, pois ainda carece de laboratórios acreditados pelo INMETRO para prestar serviços para a indústria de equipamentos elétricos em Alta Tensão em Corrente Contínua (HVDC). Deve-se considerar, ainda, que novos investimentos na geração e transmissão em larga escala de energia mobilizam a indústria e geram demanda para ensaios em novos equipamentos que serão instalados. Para realizar esses ensaios, de forma a garantir exatidão, confiabilidade e rastreabilidade, é necessária uma estrutura metrológica em Alta Tensão, ou seja, é preciso criar padrões e incentivar a acreditação de laboratórios. Nesse sentido o objetivo desta dissertação foi à construção de um protótipo de Divisor para Alta Tensão (DVAT) do tipo resistivo de alta impedância, para medição da grandeza tensão em corrente contínua acima de $1 \mathrm{kV}$. O padrão foi construído para ser utilizado na faixa de 1 $\mathrm{kV}$ a $50 \mathrm{kV}$ e será calibrado em baixa tensão rastreado à RBC. Em alta tensão será o elo na cadeia de rastreabilidade entre o padrão do INMETRO e os divisores dos laboratórios industriais e também na calibração de padrões de trabalho para HVDC na faixa de $1 \mathrm{kV}$ a $250 \mathrm{kV}$.

\section{Palavras-chave}

Metrologia; Qualidade; Padrão; Rastreabilidade; Confiabilidade; Corrente Contínua; HVDC; Calibração; Ensaios. 


\section{Abstract}

Fernandes da Silva, Márcio Thelio; Barbosa, Carlos Roberto Hall; (Advisor). Design \& Development of a Standard for High Voltage in Direct Current in the Range of $1 \mathbf{~ k V}$ to $50 \mathrm{kV}$. Rio de Janeiro, 2011. 84p. MSc. Disertation - Programa de Pós-Graduação em Metrologia, Pontifícia Universidade Católica do Rio de Janeiro.

Metrology and quality are closely related and constitute a powerful tool for transforming the technological infrastructure of countries and organizations. The advancement of scientific and technological development is strongly grounded in the advancement of metrology. The National Institute of Metrology, Standardization and Industrial Quality (INMETRO) is the highest organism responsible by the conservation and maintenance of base and derived units of the International System of Units (SI) in the country. The quantity voltage, up to 1 $\mathrm{kV}$, is traced to national standards in the Brazilian Calibration Network (RBC), but for voltages above $1 \mathrm{kV}$ there is no laboratory in the country accredited by INMETRO to provide services for the electrical equipment industry in HVDC. It should also be considered that new investments in generation and transmission of large scale energy mobilize the industry and generates demand for testing of new equipment. To perform these tests, so as to ensure reliability, accuracy and traceability, a metrology structure is needed in high voltage, so it is necessary to create standards and encourage the accreditation of laboratories. In this sense the main purpose of this dissertation was to build a prototype for a standard High Voltage Divider (DVAT) of the high impedance resistive type for measuring the quantity voltage above $1 \mathrm{kV}$. The standard was designed for use in the range of $1 \mathrm{kV}$ to $50 \mathrm{kV}$ and will be calibrated in low voltages and traceable to the RBC. In the high voltage range, it will be the link in the chain of traceability between INMETRO standards and the dividers of industrial laboratories, and also to calibrate other working standards for HVDC, especially in the range of $1 \mathrm{kV}$ to $250 \mathrm{kV}$.

\section{Keywords}

Metrology; Quality; Standard; Traceability; Reliability; HVDC; Calibration; Testing. 


\section{Sumário}

1 Introdução 14

$\begin{array}{ll}\text { 1.1. Objetivo } & 16\end{array}$

1.2. Conceitos básicos 18

1.3. Estrutura da dissertação 19

2 Padrões mundiais para medição de HVDC 21

2.1. Histórico da grandeza tensão 21

2.1.1. A Pilha de Volta 22

2.1.2. O Sistema Internacional de Unidades (SI) 23

2.1.3. Efeito Josephson 25

2.2. Conceitos sobre sistemas de medição 28

2.2.1. Sistema de Medição (SM) 28

2.2.2. Divisor de tensão 29

2.3. Padrões para tensão contínua (CC ou DC) 30

2.3.1. Baixa tensão em corrente contínua (BTCC) 30

2.3.2. Alta tensão em corrente contínua (ATCC ou HVDC) 31

2.4. Padrões para tensão alternada (CA) 32

2.4.1. Baixa tensão em corrente alternada (BTCA) 32

2.4.2. Alta tensão em corrente alternada (ATCA ou HVAC) 33

2.4.3. Divisores de tensão 33

2.5. Normalização em alta tensão 36

2.6. Equipamentos comerciais 37

$\begin{array}{ll}\text { 2.7. Laboratórios e rastreabilidade em AT } & 37\end{array}$

3 Aspectos gerais da transmissão em CC e CA 40

3.1. Interligações de sistemas elétricos através de elos HVDC 42

3.2. Vantagens do HVDC 44

3.3. Transmissão de energia em grande escala 46

3.4. Novos projetos para expansão da transmissão em HVDC no Brasil 48

3.5. Tendências tecnológicas 52 
4 Caracterização do projeto $\quad 57$

4.1. Seleção dos resistores $\quad 59$

4.2. Avaliação da estabilidade com a temperatura e tensão 60

4.3. Construção do divisor 62

5 Avaliação de desempenho do divisor 66

5.1. Avaliação de desempenho 66

5.1.1. Corrente de fuga 66

5.1.2. Descarga Parcial $\quad 67$

5.1.3. Termovisão 67

5.1.4. Tensão aplicada 68

5.1.5. Avaliação de desempenho dinâmico 68

5.2. Incerteza de medição 69

5.2.1. Baixa tensão do divisor 70

5.2.2. Medição do Fator de Escala (FE) do divisor $\quad 71$

5.2.3. Influência da temperatura (CT) 71

5.2.4. Influência da tensão (CV) 71

5.2.5. Corrente de fuga 72

5.2.6. Descarga Parcial 72

5.2.7. Estabilidade a curto prazo 72

5.2.8. Linearidade e comparação com INMETRO 72

5.2.9. Graus de liberdade efetivos 73

5.2.10. Incerteza Combinada de Medição 73

6 Conclusões e recomendações $\quad 75$

Referências bibliográficas $\quad 82$ 


\section{Lista de figuras}

Figura1 - Faixa da grandeza tensão em CC na RBC limitada em torno de $1 \mathrm{kV} \quad 14$

Figura 2 - Diagrama elétrico básico do sistema padrão de medição até $50 \mathrm{kV} \quad 17$

Figura 3 - A pilha de Volta 23

Figura 4 - Unidades de base do SI. Fonte: T. J. Queen (BIPM) 24

Figura 5 - Grau de incerteza na medição do volt ao longo de 7 décadas 25

Figura 6 - Características VxI para 3 junções diferentes e $n_{i}=1$

Figura 7 - "Chip" das junções Josephson em tamanho real 27

Figura 8 - Componentes básicos de um sistema de medição utilizado em alta tensão 28

Figura 9 - Circuitos simplificados de diferentes Divisores de Alta Tensão (DVAT) 29

Figura 10 - Conservação do padrão de tensão do INMETRO 30

Figura 11 - Conjunto de pilhas eletrônicas padrão do INMETRO de $1,018 \mathrm{~V}$ e $10 \mathrm{~V} \quad 31$

Figura 12 - Dispositivos de conversão para medição de tensão em HVDC 31

Figura 13 - Divisor padrão do INMETRO para HVDC até $150 \mathrm{kV}$

Figura 14 - Instrumentos utilizados na transferência do padrão CC para CA 33

Figura 15 - Representação simplificada de um divisor de tensão resistivo 34

Figura 16 - Divisor padrão do INMETRO (divisor vermelho) para ATCA de 1-200 kV 35

Figura 17 - Cadeia de rastreabilidade para HVDC 39

Figura 18 - Cadeia de rastreabilidade para ATCA 39

Figura 19 - Opções típicas de transmissão em HVDC e ATCA 42

Figura 20 - Comparação de custos para a transmissão em HVDC e ATCA 45

Figura 21 - Ilustração do sistema de geração e transmissão de energia de ITAIPU 47

Figura 22 - Investimentos e projetos da expansão da transmissão até 2015

Figura 23 - Projetos da expansão da geração e transmissão para Belo Monte 51

Figura 24 - Projetos da expansão da geração e transmissão para o Madeira 52

Figura 25 - Diagrama elétrico básico do padrão para HVDC 58

Figura 26 - Cadeia de rastreabilidade para HVDC 59

Figura 27 - Avaliação do coeficiente térmico dos resistores de alta e baixa tensão $\quad 60$

Figura 28 - Avaliação do resistor RHV com variação da tensão 61

Figura 29 - Avaliação do resistor RLV com variação da tensão 61

Figura 30 - Distribuição das linhas de campo elétrico ao longo do divisor 63 
Figura 31 - Cálculo da distribuição das linhas de campo elétrico ao longo do divisor 63

Figura 32 - Detalhes da estrutura com as dimensões do divisor 64

Figura 33 - Corte "A-A" com a vista da base do divisor 65

Figura 34 - Foto do divisor para HVDC até $50 \mathrm{kV} \quad 65$

Figura 35 - Descargas parciais no padrão na tensão alternada de 35,4 kV 67

Figura 36 - Ensaio de termovisão no padrão em 50 kV em corrente contínua 68

Figura 37 - Resposta ao degrau do padrão para HVDC 69

Figura 38 - Diagrama para identificação das fontes de incerteza 69

Figura 39 - Linearidade do padrão para HVDC até 50 kV 73

Figura 40 - Comparação de custos para a transmissão em HVDC e ATCA 76

Figura 41 - Cadeia de rastreabilidade para HVDC $\quad 77$ 


\section{Lista de Tabelas}

Tabela 1 - Especificação básica de calibradores comerciais multifunção............................ 37

Tabela 2 - Lista de laboratórios acreditados para ATCA …................................................. 38

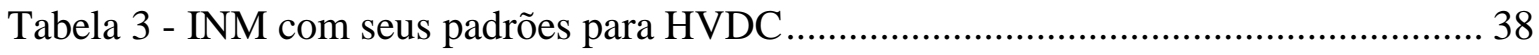

Tabela 4 - Níveis de tensão e potência para ATCA …....................................................... 45

Tabela 5 - Comparação entre HVDC e ATCA em ITAIPU ................................................ 45

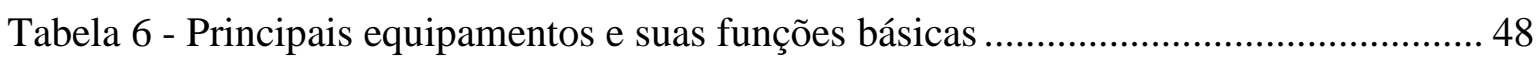

Tabela 7 - Dados obtidos na avaliação do divisor ............................................................ 70

Tabela 8 - Resultados da estatística descritiva ................................................................. 70

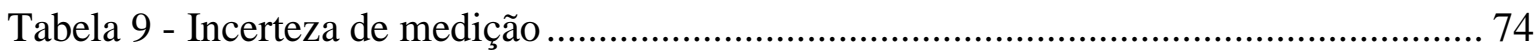

Tabela 10 - Incerteza de medição considerando comportamento linear do padrão.............. 78

Tabela 11 - Laboratórios acreditados pelo INMETRO para ATCA …................................ 80 